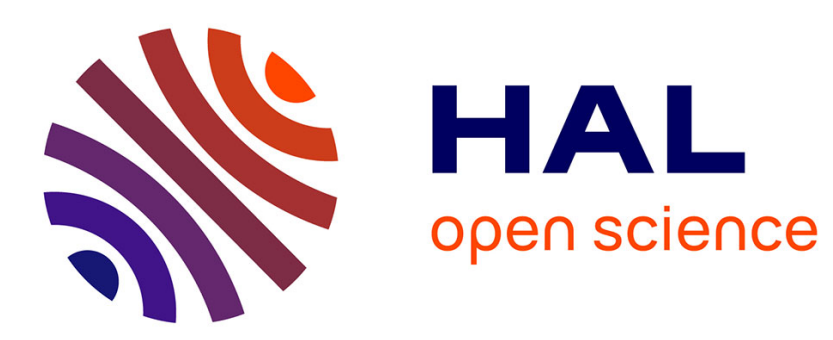

\title{
A framework for robot learning during child-robot interaction with human engagement as reward signal
}

\author{
Mehdi Khamassi, G Chalvatzaki, T Tsitsimis, G Velentzas, C Tzafestas
}

\section{To cite this version:}

Mehdi Khamassi, G Chalvatzaki, T Tsitsimis, G Velentzas, C Tzafestas. A framework for robot learning during child-robot interaction with human engagement as reward signal. 27th IEEE International Symposium on Robot and Human Interactive Communication (RO-MAN 2018), Aug 2018, Nanjing,

China. hal-02324150

\author{
HAL Id: hal-02324150 \\ https://hal.science/hal-02324150
}

Submitted on 21 Oct 2019

HAL is a multi-disciplinary open access archive for the deposit and dissemination of scientific research documents, whether they are published or not. The documents may come from teaching and research institutions in France or abroad, or from public or private research centers.
L'archive ouverte pluridisciplinaire HAL, est destinée au dépôt et à la diffusion de documents scientifiques de niveau recherche, publiés ou non, émanant des établissements d'enseignement et de recherche français ou étrangers, des laboratoires publics ou privés. 


\title{
A framework for robot learning during child-robot interaction with human engagement as reward signal
}

\author{
M. Khamassi ${ }^{1,2}$ and G. Chalvatzaki ${ }^{1}$ and T. Tsitsimis ${ }^{1}$ and G. Velentzas ${ }^{1}$ and C. Tzafestas ${ }^{1,3}$
}

\begin{abstract}
Using robots as therapeutic or educational tools for children with autism requires robots to be able to adapt their behavior specifically for each child with whom they interact. In particular, some children may like to be looked into the eyes by the robot while some may not. Some may like a robot with an extroverted behavior while others may prefer a more introverted behavior. Here we present an algorithm to adapt the robot's expressivity parameters of action (mutual gaze duration, hand movement expressivity) in an online manner during the interaction. The reward signal used for learning is based on an estimation of the child's mutual engagement with the robot, measured through non-verbal cues such as the child's gaze and distance from the robot. We first present a pilot joint attention task where children with autism interact with a robot whose level of expressivity is pre-determined to progressively increase, and show results suggesting the need for online adaptation of expressivity. We then present the proposed learning algorithm and some promising simulations in the same task. Altogether, these results suggest a way to enable robot learning based on non-verbal cues and to cope with the high degree of nonstationarities that can occur during interaction with children.
\end{abstract}

Keywords: HRI, Reinforcement Learning, Active Exploration, Autonomous Robotics, Engagement, Joint Action.

\section{INTRODUCTION}

In this short paper, we present recent progresses in developing robot learning abilities for the adaptation to humanspecific requirements during child-robot interaction. In particular, we aim at enabling the robot to vary the level of expressivity of its actions in order to increase the child's mutual engagement with the robot and thus contribute to further develop children's social interaction skills. Mutual engagement can be defined as "the process by which interactors start, maintain and end their perceived connection to each other during an interaction" [1].

Researches in the field of social robotics have recently shown a growing interest in monitoring human and robot gaze during social interaction [2], [3]. Results show that gaze following improves intention readout, efficiency of joint action, and arouses on human partners the illusion of a social intelligence. Conversely, it has been proposed that monitoring the level of engagement of the human during the task, for instance through the monitoring of body posture and gaze, may provide the robot with crucial information to assess how it is perceived by the human, how this perception changes according to the behaviors shown by the social

\footnotetext{
${ }^{1}$ All authors are with the School of Electrical and Computer Engineering, National Technical University of Athens, Greece.

${ }^{2}$ Mehdi Khamassi is also with Sorbonne Université, CNRS, Institute of Intelligent Systems and Robotics, Paris, France.

${ }^{3}$ Correspondence: ktzaf@cs.ntua.gr
}

robot, and hence to improve the quality of human-robot interaction [4]. However, to our knowledge no one has yet proposed a way to make the robot learn on the fly in response to changes in human engagement. Previous researches having applied reinforcement learning to human-robot interaction have most of the time employed discrete action spaces (e.g. [5]), hence preventing generalization to more complex tasks requiring continuous motor actions.

\section{PILOT CHILD-ROBOT INTERACTION STUDY WiTH CHILDREN WITH AUTISM}

The general experimental paradigm adopted here consists in having a small humanoid robot interact with children (one at a time), under the supervision of an observing human adult, and finding the appropriate robot behavior to maximize children's engagement in the task. This paradigm follows the objectives defined in the framework of the EU-funded project BabyRobot (H2020-ICT-24-2015-6878310), where a set of child-robot interaction use-cases have been designed and implemented to study the development of specific socioaffective, communication and collaborative skills in typically developing children as well as children with Autistic Spectrum Disorders (ASD). In this framework, we have set up a pilot experiment ${ }^{1}$ where the NAO robot is interacting with a child (Fig. 1), and repeatedly points at an unreachable object while varying the level of expressivity of its pointing gesture (i.e., opening-and-closing hand for a certain duration, bending its torso with a certain angle in the direction of the object, gazing at the child for a certain duration) until the child understands the "intention" of the robot and engages himself/herself into joint action in order to help the robot grasp the object. The engagement estimation, in this pilot study, was provided in real-time by an expert who observed the child during the interaction with the robot, considering five discrete levels of engagement ( 0 to 4 , with 0 meaning absence of engagement and 4 meaning full engagement and attempt to offer help).

We present here some preliminary results for this real HRI task for which we have yet performed the experiment only with a small number of children with mild and moderate ASD symptoms, plus a few children with severe symptoms (12 children in total so far). First, children with severe symptoms expressed no interest in the task, neither in the condition with the robot nor in a control condition where the child interacts with a human expert rather than with the

\footnotetext{
${ }^{1}$ This experiment has been approved by the ethical committee of Athena Research Center, Greece. The children's parents provided written consents
} 


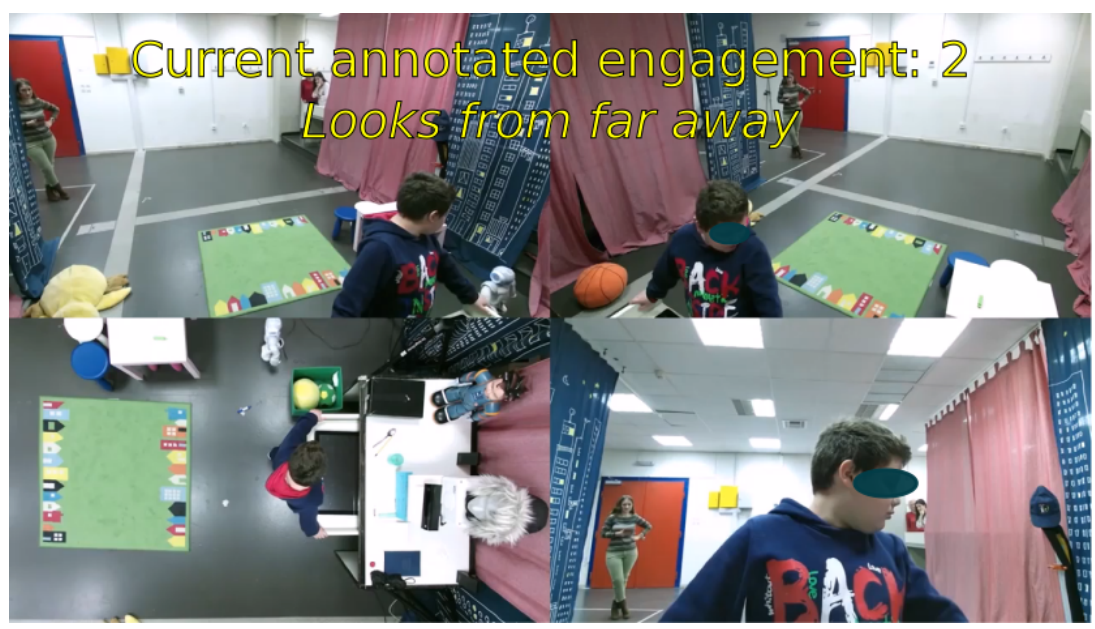

Fig. 1. Pilot child-robot interaction study with children with ASD. The figure shows a moment where a child with ASD showed moderate engagement while the robot moved its arm up and down to point at an object on a table.

robot. In contrast, children with mild symptoms displayed great enthusiasm and interest in playing with the robot as well as with the researcher and enjoyed the whole process. These children were able to respond quite well to the task and completed the experiment with success. Overall, we found that two out of eight children with mild symptoms successfully maximized their engagement in joint attention with the robot and gave the object to the robot spontaneously. The remaining six children successfully increased their engagement, although not optimally, ending up moving the object closer to the robot but not handing it in. The two children with moderate symptoms also increased engagement and ended up exploring the object pointed at by the robot. Finally, again children with severe symptoms did not respond to the task.

Figure 1 shows one child performing the task, looking at the NAO robot (moderate engagement) while the latter moved its arm down after pointing at the object on the small white table. The psychologist who can be seen near the red door is manually annotating the child's engagement so that the robot can adapt its behavior. These results are promising and stimulating in that eight children that we interviewed after the task said that they would like to play more often with the robot and that they found the tasks we proposed them relatively easy. But many more subjects for each level of severity of ASD symptoms are required before allowing some statistics on the results. Interestingly, studying how the robot's movements affected the child's engagement, we observed that when the robot opened and closed its grip or exchanged glances between the child and the object for a period of time while pointing at the object, it contributed to an increase in the child's engagement. This suggests that varying the level of expressivity in the robot's actions in time was key to increase child engagement. Nevertheless, different levels of expressivity appeared to be appropriate for different children. It is thus relevant to propose a way for the robot to autonomously learn the appropriate degree of expressivity appropriate for each child.

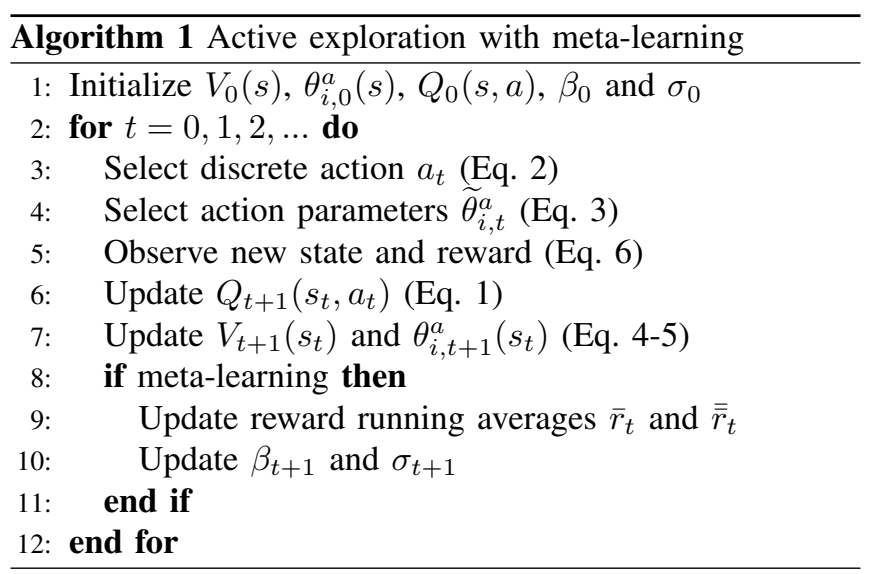

\section{ROBOT LEARNING ALGORITHM}

The proposed algorithm is summarised in Algorithm 1. It is based on reinforcement learning with parameterized action spaces [6], [7]. It employs a set of discrete actions $A_{d}=\left\{a_{1}, a_{2}, \ldots, a_{k}\right\}$, where each action $a \in A_{d}$ features $m_{a}$ continuous parameters $\left\{\theta_{1}^{a}, \ldots, \theta_{m_{a}}^{a}\right\} \in \mathbb{R}^{m_{a}}$, which enables to benefit from the simplicity of task decomposition into a small set of discrete actions while at the same time being able to exploit the precision of continuous motor execution. Learning the value of discrete action $a_{t} \in A_{d}$ selected at timestep $t$ in state $s_{t}$ is done through Q-Learning [8]:

$\Delta Q_{t}\left(s_{t}, a_{t}\right)=\alpha_{Q}\left(r_{t}+\gamma \max _{a}\left(Q_{t}\left(s_{t+1}, a\right)\right)-Q_{t}\left(s_{t}, a_{t}\right)\right)$

where $\alpha_{Q}$ is a learning rate and $\gamma$ is a discount factor. The probability of executing discrete action $a_{j}$ at timestep $t$ is given by a Boltzmann softmax equation:

$$
P\left(a \mid s_{t}, \beta_{t}\right)=\frac{\exp \left(\beta_{t} Q_{t}\left(s_{t}, a\right)\right)}{\sum_{a^{\prime}} \exp \left(\beta_{t} Q_{t}\left(s_{t}, a^{\prime}\right)\right)}
$$


where $\beta_{t}$ is a dynamic inverse temperature meta-parameter which will be tuned through meta-learning (see below).

In parallel, continuous parameters $\widetilde{\theta}_{i, t}^{a}$ with which action $a$ is executed at timestep $t$ are selected from a Gaussian exploration function centered at the current values $\theta_{i, t}^{a}\left(s_{t}\right)$ in state $s_{t}$ of the parameters of this action:

$$
P\left(\widetilde{\theta}_{i, t}^{a} \mid s_{t}, a_{t}, \sigma_{t}\right)=\frac{1}{\sqrt{2 \pi} \sigma_{t}} \exp \left(-\left(\widetilde{\theta}_{i, t}^{a}-\theta_{i, t}^{a}\left(s_{t}\right)\right)^{2} /\left(2 \sigma_{t}^{2}\right)\right)
$$

where the width $\sigma_{t}$ of the Gaussian is tuned through meta-learning (see below) and continuous action parameters $\theta_{i, t}^{a}\left(s_{t}\right)$ are learned with the CACLA algorithm [9]. A reward prediction error is computed from the critic: $\delta_{t}=r_{t}+$ $\gamma V_{t}\left(s_{t+1}\right)-V_{t}\left(s_{t}\right)$ and is used to update the critic and the actor:

$$
\begin{gathered}
V_{t+1}\left(s_{t}\right)=V_{t}\left(s_{t}\right)+\alpha_{C} \delta_{t} \\
\theta_{i, t+1}^{a}\left(s_{t}\right)=\theta_{i, t}^{a}\left(s_{t}\right)+\alpha_{A} \delta_{t}\left(\widetilde{\theta}_{i, t}^{a}-\theta_{i, t}^{a}\left(s_{t}\right)\right)
\end{gathered}
$$

where $\alpha_{C}$ and $\alpha_{A}$ are learning rates.

In order to perform active exploration, we apply a noiseless version of the meta-learning algorithm of [10], which tracks online variations of the agent's performance measured by short-term $\bar{r}_{t}$ and long-term $\overline{\bar{r}}_{t}$ reward running averages. At each timestep, we use the difference between the two averages to simultaneously tune the inverse temperature $\beta_{t}$ used for selecting between discrete actions $a$, and the width $\sigma_{t}$ of the Gaussian distribution from which each continuous action parameter $\theta_{i}^{a}$ is sampled around its current value. The main idea is that when the performance is better than average, exploration should be decreased in order to reach optimality levels. In contrast, sudden drops in the performance should lead to increases in exploration in order to adapt to environmental non-stationarities.

Finally, we need to define a reward function for humanrobot interaction tasks. This is not an easy task since during interaction the actions performed by a robot may have delayed effects on the human's behavior and on his engagement. To mimic this, we chose a reward component to be given by a dynamical system which is based on the virtual engagement $E$ of the human in the task. In our simulations, the quantified engagement arbitrarily starts at 5, increases up to a maximum $E_{M}=10$ when the robot performs the appropriate actions with the appropriate parameters, and decreases down to a minimum $E_{m}=0$ otherwise:

$$
E_{t+1}= \begin{cases}E_{t}+\eta_{1}\left(E_{M}-E_{t}\right) H\left(\theta_{t}^{a}\right), & \text { if } a_{t}=a^{\star} \& H\left(\theta_{t}^{a}\right) \geq 0 \\ E_{t}-\eta_{2}\left(E_{m}-E_{t}\right) H\left(\theta_{t}^{a}\right), & \text { if } a_{t}=a^{\star} \& H\left(\theta_{t}^{a}\right)<0 \\ E_{t}+\eta_{2}\left(E_{m}-E_{t}\right), & \text { otherwise }\end{cases}
$$

where $\eta_{1}=0.1$ is the increasing rate, $\eta_{2}=0.05$ is the decreasing rate, and $\mathcal{H}(x)$ is the reengagement function given by $\mathcal{H}(x)=2\left(\exp \left(-\frac{\left(x-\mu^{\star}\right)^{2}}{2 \sigma^{\star 2}}\right)-0.5\right)$ where $a^{\star}, \mu^{\star}$ and $\sigma^{\star}$ are respectively the optimal action, action parameter and variance around $a^{\star}$.

The reward function is then computed as $r_{t+1}=E_{t+1}+$ $\lambda \Delta E_{t}$ where $\lambda=0.7$ is a weight and $\Delta E_{t}=E_{t+1}-$ $E_{t}$. This reward function ensures that the algorithm gets rewarded in cases where the engagement $E_{t+1}$ is low but nevertheless has just been increased by the action n-tuple $\left(a_{t}, \theta_{1, t}^{a}, \theta_{2, t}^{a}, \ldots, \theta_{m_{a}, t}^{a}\right)$ performed by the robot.

\section{Simulations}

We performed numerical simulations of the learning algorithm in a task identical to the child-robot interaction pilot task described above. We implemented the simulations in the virtual robot experimentation platform (V-REP). In the considered scenario, the NAO robot points at an object on a table with different degrees of action expressivity so as to catch the child's attention and thus increase mutual engagement (Fig.2(b)). We parameterized the simulated pointing action of the robot with two parameters $\left(t_{1}, t_{2}\right)$ corresponding to the time in seconds the robot would spend iteratively opening-closing its hand during pointing, and the time spent exchanging glances with the child. Examples of different levels of expressivity defined by these parameters are shown in Table I.

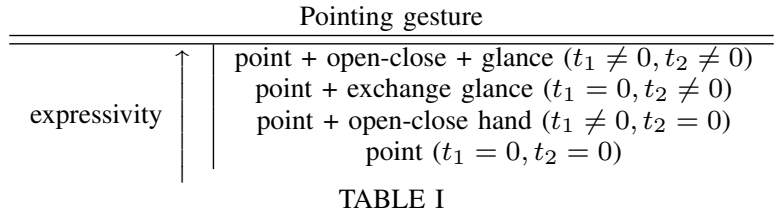

ROBOT'S POINTING ACTION WITH PARAMETERS CORRESPONDING TO INCREASING LEVELS OF EXPRESSIVITY.

We initialized the algorithm based on the parameters obtained on average during previous interactions with simulated children. This way, the algorithm started from a meaningful average value of action parameters/durations $\left(\overline{t_{1}}, \overline{t_{2}}\right)$, rather than being initialized randomly, and then adapted to each specific child. We defined a time range from 0 to 10 seconds. Fig. 2(b) shows the average performance over 10 simulations. The robot firstly interacted with an "average child", meaning that the child engaged optimally with parameters $\left(\overline{t_{1}}, \overline{t_{2}}\right)$. Then, at timestep 40, the experiment involved another child (child 1) with different optimal parameters. The engagement of child 1 was initially low but progressively re-increased as the robot was finding the optimal continuous action parameters. The figure also illustrated the increased variance in executed action parameters during exploration followed by a re-focus around the learned parameters during exploitation. Similarly, at timestep 80 child 2 took the place of child 1 and the robot readjusted its parameters. Importantly, we observe that in less than 10 timesteps the robot found the optimal parameter values for the different children whose engagement reached 8 in just a few timesteps. This thus illustrates a sufficiently fast adaptation process to work online during real child-robot interactions. 


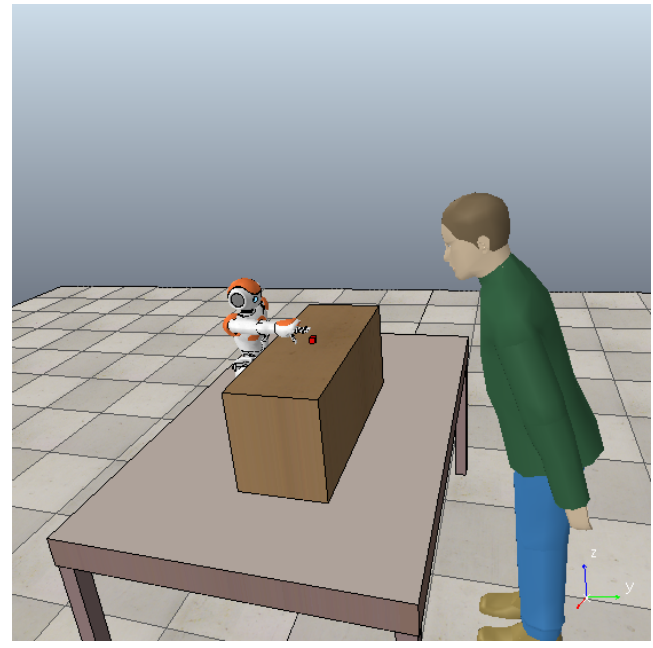

(a) V/REP SIMULATION ENVIRONMENT
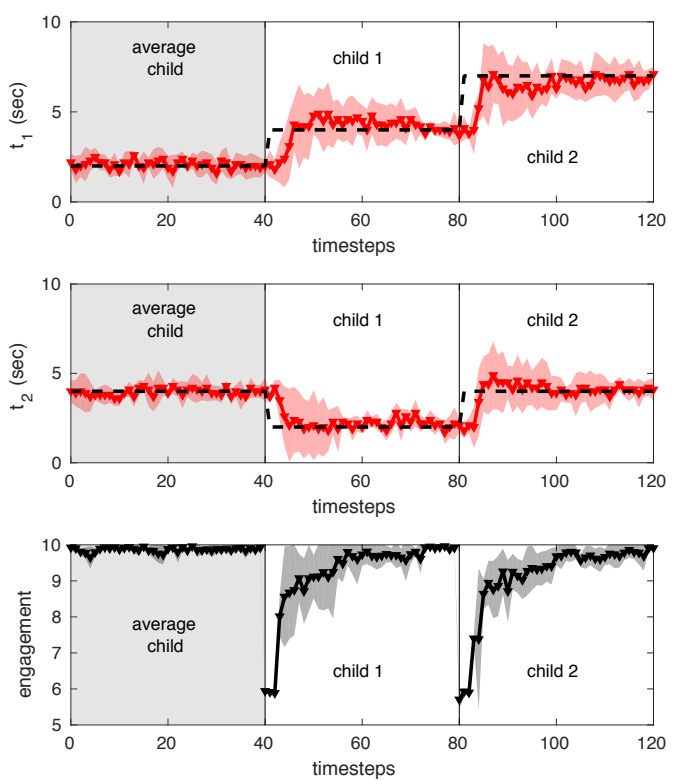

(b) SIMULATION RESULTS

Fig. 2. Numerical simulations. (a) Setup used for the simulations of the same task as the pilot real child-robot interaction experiment. (b) Simulation results. Left: Before timestep 0 the robot executed the default parameters values, no adaptation was performed. After timestep 0 , the robot adapted its action parameters (black) towards the optimal action parameters (red). Right: Child's engagement reached $90 \%$ within less than 10 trials.

\section{CONCLUSIONS AND FUTURE WORK}

In this short paper, we presented recent progresses in developing robot learning abilities for the adaptation to human-specific requirements during child-robot interaction. In particular, we aimed at enabling the robot to vary the level of expressivity of its actions in order to increase the child's mutual engagement with the robot and thus contribute to further develop children's social interaction skills. We first showed some preliminary results in a pilot study involving a robot with a predetermined sequence of increased expressivity of action while pointing at an unreachable object until a child with ASD understands that the robot needs help and engages in joint action. The preliminary results suggest that the level of expressivity does play a role in engaging the child, but should nevertheless be adapted through online learning to each interacting child. We then presented a learning algorithm based on reinforcement learning in parameterized action spaces [6], [7] - to benefit from the simplicity of task decomposition into a small set of discrete actions while at the same time being able to exploit the precision of continuous motor execution - to which we added active exploration so as to cope with the frequent nonstationarities that can occur during human-robot interaction. We presented simulation results showing that the algorithm can adapt in a sufficiently small number of trials to be applied to adaptation in real-time during interaction.

In future work, we plan to test the learning algorithm during real child-robot interaction. We moreover plan to study whether the average parameters over different interacting children is efficient or whether there exists distinct clusters of parameters - especially within the data obtained in the real experiments - that should be used as separate initialization points for the learning algorithm.

\section{ACKNOWLEDGEMENTS}

The authors would like to thank psychologists Christina Papaeliou and Asimenia Papoulidi, and the Special Elementary School for Children with ASD of Piraeus, Greece, for enabling us to make these experiments with children with ASD. This research work has been partially supported by the EU-funded Project BabyRobot (H2020-ICT-24-2015, grant agreement no. 687831), and by the Centre National de la Recherche Scientifique (MI ROBAUTISTE \& PICS 279521).

\section{REFERENCES}

[1] C. L. Sidner, C. Lee, C. D. Kidd, N. Lesh, and C. Rich, "Explorations in engagement for humans and robots," Artificial Intelligence, vol. 166, no. 1-2, pp. 140-164, 2005.

[2] J.-D. Boucher, U. Pattacini, A. Lelong, G. Bailly, F. Elisei, S. Fagel, P. F. Dominey, and J. Ventre-Dominey, "I reach faster when i see you look: gaze effects in human-human and human-robot face-to-face cooperation," Frontiers in neurorobotics, vol. 6, 2012.

[3] S. Bampatzia, V. Vouloutsi, K. Grechuta, S. Lallée, and P. F. Verschure, "Effects of gaze synchronization in human-robot interaction," in Conference on Biomimetic and Biohybrid Systems. Springer, 2014, pp. 370-373.

[4] S. M. Anzalone, S. Boucenna, S. Ivaldi, and M. Chetouani, "Evaluating the engagement with social robots," International Journal of Social Robotics, vol. 7, no. 4, pp. 465-478, 2015.

[5] M. Khamassi, S. Lallée, P. Enel, E. Procyk, and P. Dominey, "Robot cognitive control with a neurophysiologically inspired reinforcement learning model," Frontiers in Neurorobotics, vol. 5:1, 2011.

[6] W. Masson and G. Konidaris, "Reinforcement learning with parameterized actions," in Proceedings of the Thirtieth AAAI Conference on Artificial Intelligence (AAAI-16), 2016.

[7] M. Hausknecht and P. Stone, "Deep reinforcement learning in parameterized action space," in International Conference on Learning Representations (ICLR 2016), 2016.

[8] C. Watkins and P. Dayan, "Q-learning," Machine learning, vol. 8, no. 3-4, pp. 279-292, 1992.

[9] H. van Hasselt and M. Wiering, "Reinforcement learning in continuous action spaces," in IEEE Symposium on Approximate Dynamic Programming and Reinforcement Learning, 2007, pp. 272-279.

[10] N. Schweighofer and K. Doya, "Meta-learning in reinforcement learning." Neural Networks, vol. 16, no. 1, pp. 5-9, 2003. 